\title{
Observation of negative refraction of Dirac fermions in graphene
}

\section{Gil-Ho Lee ', Geon-Hyoung Park and Hu-Jong Lee ${ }^{\star}$}

\begin{abstract}
Half a century ago, Veselago' proposed 'left-handed' materials with negative permittivity and permeability, in which waves propagate with phase and group velocities in opposite directions. Significant work has been undertaken to attain this left-handed response, such as establishing a negative refractive index in so-called metamaterials, which consist of periodic sub-wavelength structures ${ }^{2-4}$. However, an electronic counterpart has not been demonstrated owing to difficulties in creating repeated structures smaller than the electronic Fermi wavelength of the order $\sim 10 \mathrm{~nm}$. Here, without needing to engineer sub-wavelength structures, we demonstrate negative refractive behaviour of Dirac fermions in graphene, exploiting its unique relativistic band structure ${ }^{5}$. Analysis of both electron focusing through an $n-p-n$ flat lens and negative refraction across $n-p$ junctions confirms left-handed behaviour in the electronic system. This approach to electronic optics is of particular relevance to the on-going efforts to develop novel quantum devices with emerging ${ }^{6}$ layered materials.
\end{abstract}

Owing to their wave nature, electrons follow the laws of optics when their mean free path and phase coherence length are larger than the system size. So far, electron optics has been demonstrated mostly in conventional two-dimensional electron gas (2DEG) systems such as interferometers or electrostatic lenses ${ }^{7}$. Graphene can provide an attractive platform for studying the unique electronic optics of Dirac fermions owing to its gapless and linear dispersion. Cheianov et al. ${ }^{5}$ proposed the interesting idea that transparent ballistic $n-p$ junctions of graphene can exhibit negative refractive behaviour with electrostatic gates providing control of local doping (Fig. 1a). This is a fundamentally different approach from the conventional one utilizing periodic sub-wavelength structures in metamaterials. In this approach, negative refraction is observed because the wavevector (k) and the group velocity $\left[\mathbf{v}_{\mathrm{g}}=\mathrm{d} E(\mathbf{k}) / \mathrm{d}(\hbar \mathbf{k})\right]$ of carriers are parallel or antiparallel to each other, depending on whether the carriers are electron-like $[E(\mathbf{k})=\hbar v k]$ or hole-like $[E(\mathbf{k})=-\hbar v k]$, respectively. When an electronic wave enters an $n-p$ junction, the tangential component of $\mathbf{v}_{\mathrm{g}}$ is reversed to conserve the tangential component of $\mathbf{k}\left(k_{\mathrm{i}} \sin \theta_{\mathrm{i}}=-k_{\mathrm{r}} \sin \theta_{\mathrm{r}}\right)$, whereas the perpendicular component of $\mathbf{v}_{\mathrm{g}}$ itself is preserved (Fig. 1b). As a consequence, the refraction follows Snell's law with a negative reflective index $n$, where

$$
n \equiv \frac{\sin \theta_{\mathrm{i}}}{\sin \theta_{\mathrm{r}}}=-\frac{k_{\mathrm{r}}}{k_{\mathrm{i}}}
$$

Here, $n$ is defined as the relative refractive index of the p-doped region compared to the $\mathrm{n}$-doped region. A vanishing bandgap, a distinct property of graphene, is an essential component of the a
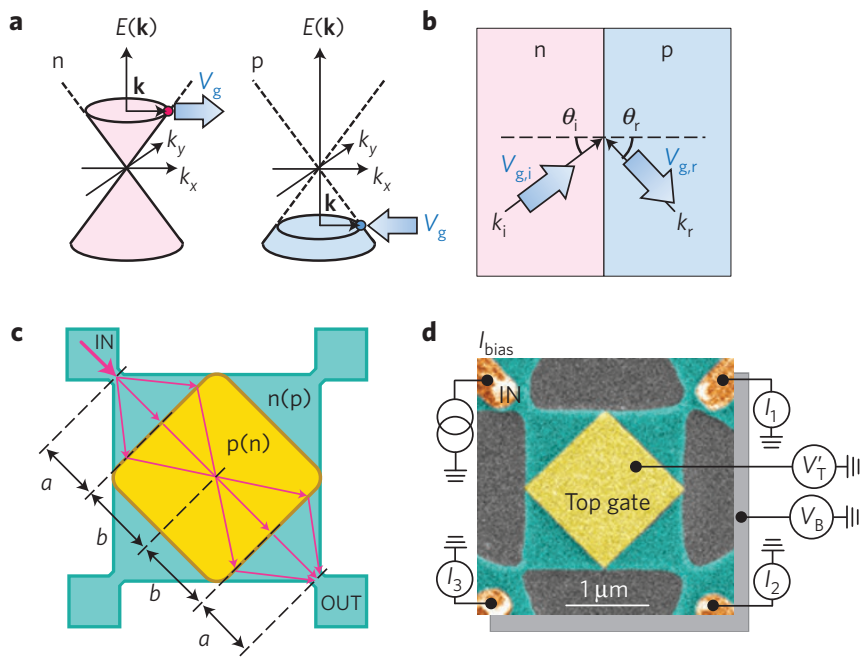

Figure 1 | Negative refraction in graphene p-n junctions. a, Band structure of graphene and the dispersion relation of the wavevector $(\mathbf{k})$ and the group velocity $\left(\mathbf{V}_{g}\right)$ for the electron-doped $(n)$ and hole-doped $(p)$ states. Red (blue) circles represent electron-(hole-)like quasiparticles. b, Negative refraction across the $n-p$ junction. $\mathbf{c}$, Schematic of Veselago lens with current trajectories under a focusing condition, where spreading electrons from the port IN are refocused on port 2. d, Scanning electron microscopy image of the Veselago lens device taken before attaching the top gate bridge connection. Orange, turquoise and yellow colours represent the $\mathrm{Cr} / \mathrm{Au}$ electrodes, the boron nitride (BN)/graphene/BN stack and the $\mathrm{Cr} / \mathrm{Au}$ top gate, respectively.

effect, because it facilitates transparent $\mathrm{p}-\mathrm{n}$ junctions for electron optics; in contrast a semiconducting $2 \mathrm{DEG}$ results in impermeable $\mathrm{p}-\mathrm{n}$ junctions due to the depletion region. Although graphene $\mathrm{p}-\mathrm{n}$ junctions have been extensively studied ${ }^{8-15}$, with clear evidence of Klein tunnelling ${ }^{16,17}$, negative refraction has not been confirmed clearly, owing either to the diffusive nature of the carriers or because the $\mathrm{p}-\mathrm{n}$ potential barrier was smoother than the electronic Fermi wavelength, $\lambda_{\mathrm{F}}(=2 \pi / k \sim \mathrm{a}$ few tens of $\mathrm{nm})$.

We fabricated a graphene-based flat lens device ${ }^{1,18}$ (or a Veselago lens) consisting of two successive $n-p$ and $p-n$ junctions, as shown in Fig. 1c,d. The constriction at each corner is analogous to a single slit in optics and ensures the injected electronic waves have a wide angular distribution, which resembles that of a Fraunhofer diffraction pattern (see Methods and Supplementary Figs 1 and 2). When the focusing condition $(n=-b / a)$ is met, electron beams from the IN port are negatively refracted successively through two $\mathrm{n}-\mathrm{p}$ and $\mathrm{p}-\mathrm{n}$ junctions, and then refocused at the OUT port. 
a
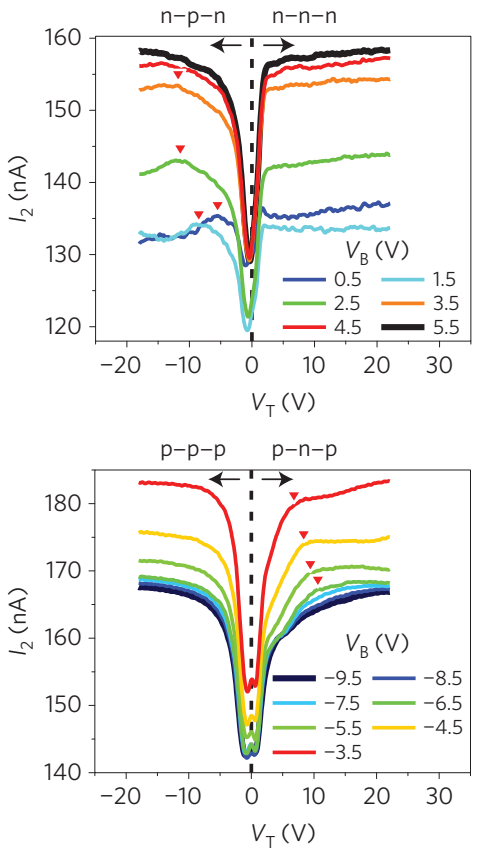

b
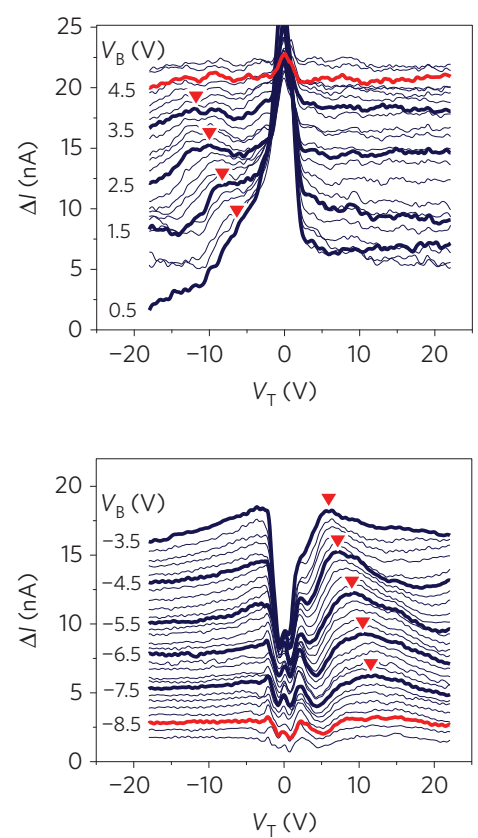

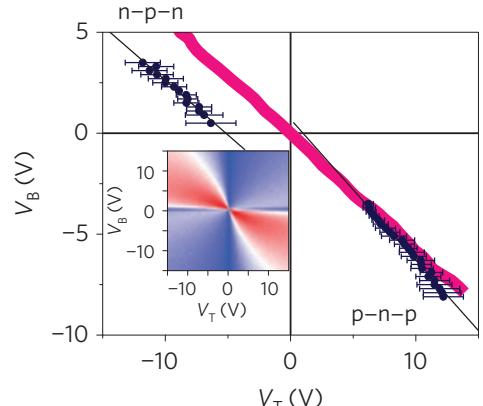

d

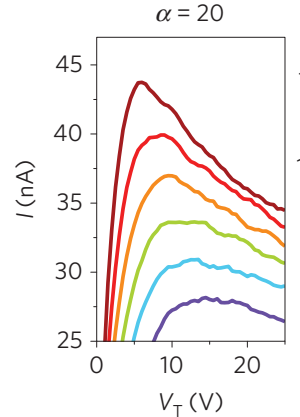

Figure 2 | Current focusing of graphene-based Veselago lens. a, $V_{T}$ dependence of $I_{2}$ with positive (upper panel) and negative (lower panel) values of $V_{B}$

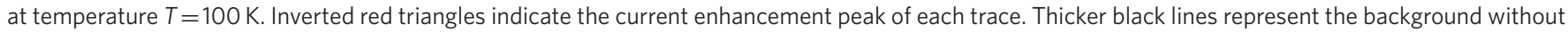
the current enhancement peak. $\mathbf{b}$, Background-subtracted current $\Delta I=I_{2}-I_{2}\left(V_{B}=5.5\right.$ for $V_{B}>0$ in the upper panel; $V_{B}=-9.5 V$ for $V_{B}<0$ in the lower panel) as a function of $V_{T}$. Traces are separated in steps of $V_{B}=0.2 \mathrm{~V}$, with an arbitrary offset for clarity. Every fifth trace is emphasized with a thicker line. Red triangles indicate the current enhancement peaks and red lines are the boundaries beyond which current enhancement vanishes. $\mathbf{c}$, Relationship

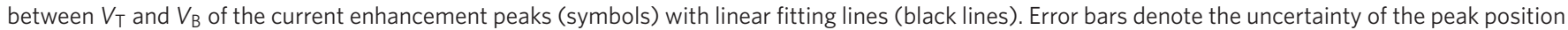
due to fluctuations of $\Delta l$. The solid red line represents the simulation result. Inset: simulated focusing current with a colour scale from blue ( 0 nA) to red

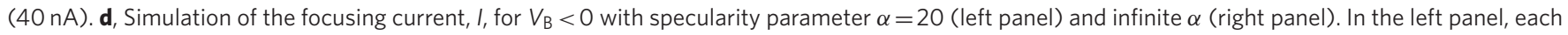
curve was offset by a step of $2 \mathrm{nA}$ from bottom to top for a clearer comparison with the lower panel of $\mathbf{b}$.

Monolayer graphene was encapsulated in insulating and atomically flat boron nitride $(\mathrm{BN})$ crystals to achieve the characteristic ballistic nature of graphene (see Methods and Supplementary Fig. 3). The top local gate voltage $\left(V_{\mathrm{T}}^{\prime}\right)$ and bottom global gate voltage $\left(V_{\mathrm{B}}\right)$ enabled in situ control of the refractive index. A thin top $\mathrm{BN}$ layer (thickness $\sim 14 \mathrm{~nm}$ ) provided sharp $\mathrm{p}-\mathrm{n}$ junctions with a characteristic sharpness of $d \sim 12 \mathrm{~nm}$ (see Methods and Supplementary Fig. 4).

The geometric asymmetry $(a=600 \mathrm{~nm}, b=720 \mathrm{~nm})$ is exaggerated in the schematic shown in Fig. 1c. Each corner with a narrow constriction (width $w \sim 100 \mathrm{~nm}$ ) was connected to the $\mathrm{Cr} / \mathrm{Au}$ metal electrode. In situ etching of the graphene just before metal deposition was implemented to form a highly transparent contact. This geometry led to a negative van der Pauw resistivity at temperature $T=4.2 \mathrm{~K}$, confirming ballistic transport. The mobility and the mean free path were estimated to be $120,000 \mathrm{~cm}^{2} \mathrm{~V}^{-1} \mathrm{~s}^{-1}$ and $1.7 \mu \mathrm{m}$ at $V_{\mathrm{B}}=V_{\mathrm{T}}=-20 \mathrm{~V}$ (see Supplementary Fig. 5). A bias current was injected $\left(I_{\text {bias }}=500 \mathrm{nA}\right)$, and the drain currents at the other three ports were measured simultaneously as a function of $V_{\mathrm{T}}^{\prime}$ and $V_{\mathrm{B}}$. The compensated top gate voltage $V_{\mathrm{T}}=\gamma V_{\mathrm{T}}^{\prime}+V_{\mathrm{B}}$, and $V_{\mathrm{B}}$ determined the carrier densities $\rho_{\mathrm{T}}=k^{2} / \pi=\eta V_{\mathrm{T}}$ and $\rho_{\mathrm{B}}=\eta V_{\mathrm{B}}$ of the top- and back-gated regions, respectively, with $\eta \sim 7.2 \times 10^{-2} \mathrm{~cm}^{-2} \mathrm{~V}^{-1}$. The ratio of top to bottom gate efficiency, $\gamma=21.0$, was determined using the values of $V_{\mathrm{T}}^{\prime}$ and $V_{\mathrm{B}}$ at the Dirac points. Independent control of $V_{\mathrm{T}}$ and $V_{\mathrm{B}}$ allowed exploration of a range of values of $n=\operatorname{sgn}\left(V_{\mathrm{T}} \cdot V_{\mathrm{B}}\right) \sqrt{V_{\mathrm{T}} / V_{\mathrm{B}}}$ for both polarities. The drain current at port $2, I_{2}$, taken at $100 \mathrm{~K}$ is plotted as a function of $V_{\mathrm{T}}$ in Fig. 2a. Near $V_{\mathrm{T}}=0$, where the top-gated region becomes most resistive, a greater current tends to flow towards ports 1 or 3 rather than port 2, resulting in a decrease of $I_{2}$ at all values of $V_{\mathrm{B}}$ (see Supplementary Fig. 6). On top of the background, each trace for $V_{\mathrm{T}}$ for different values of $V_{\mathrm{B}}$ exhibits a current focusing peak (red triangles) only in the bipolar regimes of $n-p-n$ and $\mathrm{p}-\mathrm{n}-\mathrm{p}$. As $V_{\mathrm{B}}$ moves away from the Dirac point, the focusing peaks become smaller and broader, and are eventually obscured by the background signal. This happens as $\lambda_{\mathrm{F}}$ becomes much shorter than $d$ for $V_{\mathrm{B}, \mathrm{T}}$ away from the Dirac point, resulting in increased electron reflection at the $\mathrm{p}-\mathrm{n}$ boundaries ${ }^{19,20}$. After subtracting the data in this regime as a background signal, the gate dependence of the peak positions and widths becomes more conspicuous (Fig. 2b). Current focusing peaks shift linearly with $V_{\mathrm{B}}$ for negative values of $V_{\mathrm{T}} / V_{\mathrm{B}}$. This confirms that they originate from electron focusing through the $\mathrm{p}-\mathrm{n}$ boundaries with a negative value of $n$, rather than any sample-specific doping inhomogeneity. The focusing condition is estimated to be $n=-b / a=-1.20$ for our device geometry. This value agrees well with the observations: $n_{\mathrm{npn}}=-1.35$ (for $\mathrm{n}-\mathrm{p}-\mathrm{n}$ ) and $n_{\text {pnp }}=-1.20$ (for $\mathrm{p}-\mathrm{n}-\mathrm{p}$ ), as shown in Fig. $2 \mathrm{c}$. The solid red line represents the numerical simulation result of a classical particle tracing with geometric parameters; $a, b, w$ and $d$ (see Methods and Supplementary Fig. 7) with the angle dependence of the transmission probability $T$ through $\mathrm{p}-\mathrm{n}$ boundaries ${ }^{20}$,

$$
T\left(n, \theta_{\mathrm{i}}\right)=\frac{\cos \theta_{\mathrm{i}} \cos \theta_{\mathrm{r}}}{\cos ^{2}\left[\left(\theta_{\mathrm{i}}+\theta_{\mathrm{r}}\right) / 2\right]} \exp \left(-\pi k_{\mathrm{F}} d \sin ^{2} \theta_{\mathrm{i}} \frac{2}{1-n}\right)
$$

and the Fermi velocity of graphene $v_{\mathrm{F}} \sim 10^{6} \mathrm{~ms}^{-1}$. For the unipolar case, the exponential term in equation (2) can be ignored because there is no Wentzel-Kramers-Brillouin (WKB) tunnelling term. The $V_{\mathrm{T}} / V_{\mathrm{B}}$ slope of the simulation result also agrees well with the data. A finite offset $(\sim-2.8 \mathrm{~V})$ in $V_{\mathrm{T}}$ for the linear fitting in the $\mathrm{n}-\mathrm{p}-\mathrm{n}$ regime is as small as the full-width at half-maximum (FWHM) of the Dirac point $(\Delta V \sim 2.5 \mathrm{~V})$, which represents the 
a
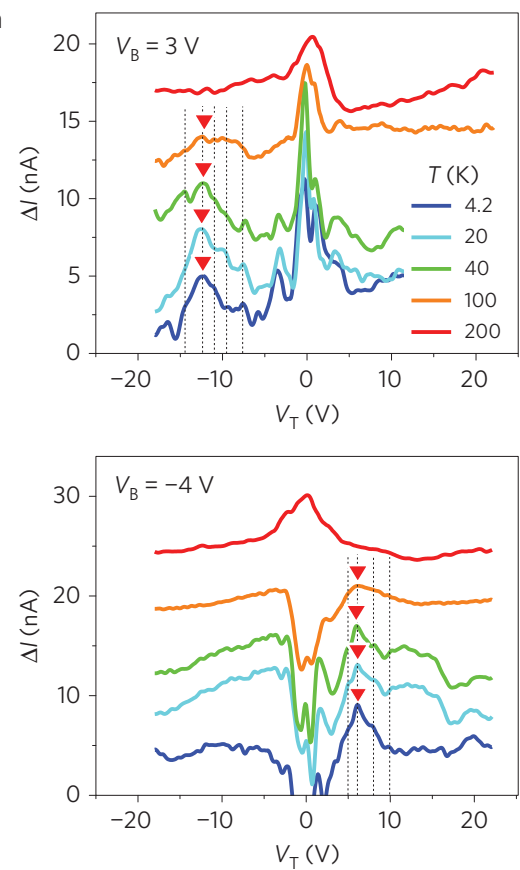

b
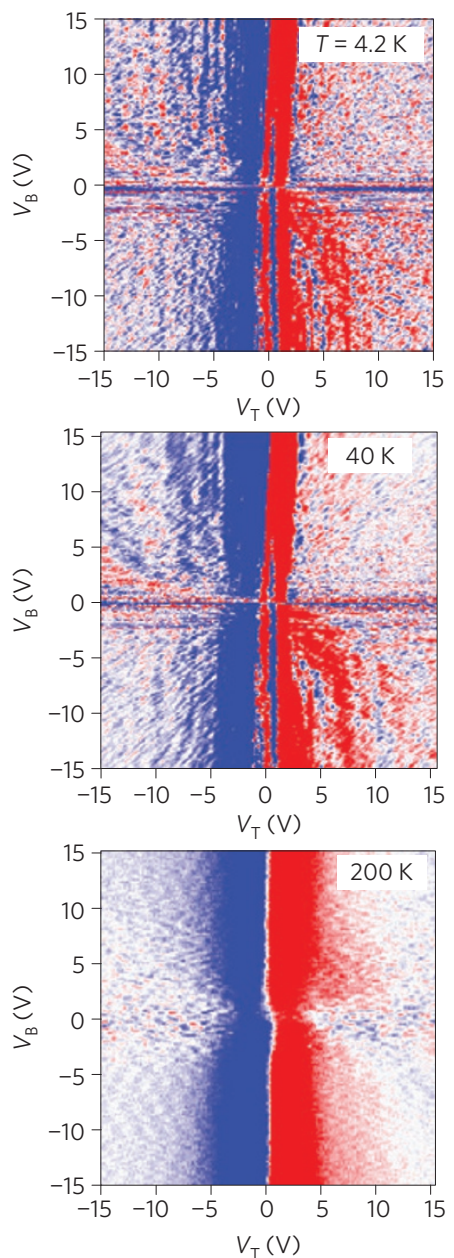
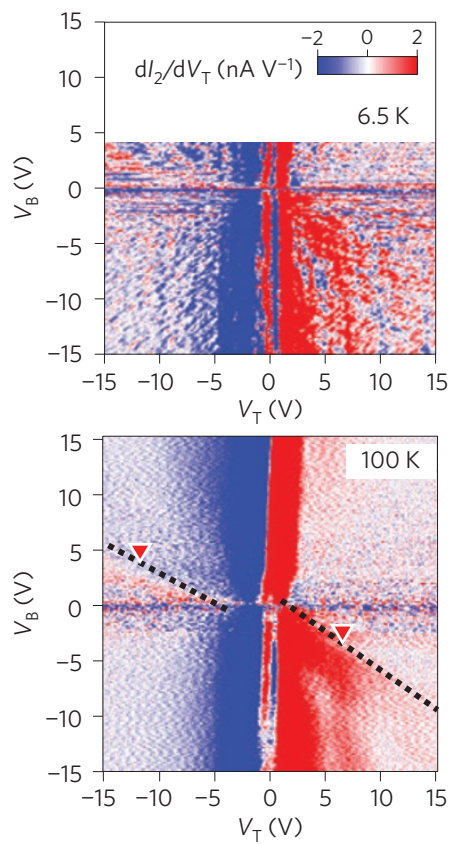

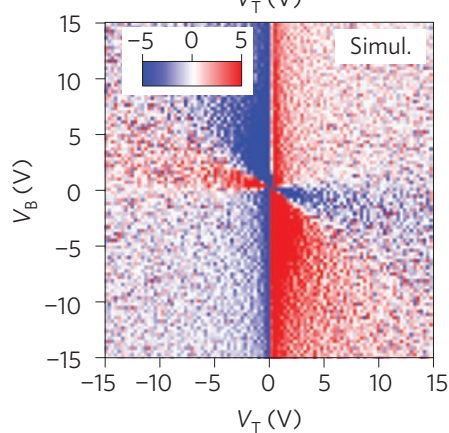

Figure 3 | Temperature dependence of current focusing. a, Background-subtracted current $\Delta /$ as a function of $V_{T}$ for $V_{B}=3 V$ (upper panel) and $V_{B}=-4 \mathrm{~V}$ (lower panel) taken at various temperatures $(T)$. Red triangles indicate the current enhancement peaks, which gradually vanish as $T$ increases. b, Colour plot of $d l_{2} / d V_{T}$ as a function of $V_{T}$ and $V_{B}$ taken at $T=4.2 \mathrm{~K}, 6.5 \mathrm{~K}, 40 \mathrm{~K}, 100 \mathrm{~K}$ and $200 \mathrm{~K}$. The last panel is the simulation result. Linear fitting lines obtained in Fig. $2 \mathrm{~d}$ are overlaid on the $T=100 \mathrm{~K}$ plot as dotted lines. They represent the boundaries between positive (red) and negative values (blue) of $d l_{2} / d V_{T}$, which correspond to the peaks of $I_{2}$.

inhomogeneity of the Fermi level. Thus, the offset may have resulted from ambiguity in the Dirac point.

The width of the current focusing peaks is related to the edge roughness of the top gate. The specularity of a $p-n$ boundary was quantified by adopting Phong's empirical model ${ }^{21}$. In this model, $\alpha$ parameterizes the specularity of the interface with a distribution probability proportional to $\cos ^{\alpha} \theta$, where the refracted beam deviates from the refraction angle of the perfect specularity case by an error angle $\theta$. In Fig. 2d, the simulation with $\alpha=20$ (FWHM of the probability distribution $\sim 30^{\circ}$ ) shows a significant resemblance to the observed results of the $\mathrm{p}-\mathrm{n}-\mathrm{p}$ case for both the width and asymmetricity of the $I$ versus $V_{\mathrm{T}}$ curves. The simulation for infinite $\alpha$ (perfect specularity, FWHM of distribution $=0^{\circ}$ ) shows much larger and sharper focusing peaks, implying that flatter $\mathrm{p}-\mathrm{n}$ boundaries can significantly improve Veselago focusing.

The temperature dependence of the Veselago focusing and the quantum interference survived up to $100 \mathrm{~K}$ and $40 \mathrm{~K}$, respectively (Fig. 3a). Temperature dependences of both features are more pronounced in a plot of the numerical derivative $\mathrm{d} I_{2} / \mathrm{d} V_{\mathrm{T}}$ (Fig. 3b). Because focusing relies on classical electron trajectories, it can persist at higher temperatures than quantum interference. A simple length scale analysis shows that the focusing signal can persist up to a temperature of the order $\hbar v_{\mathrm{F}} k_{\mathrm{F}}(w / c) \sim 170 \mathrm{~K}$, where the spread of the perpendicular component of momentum $\left(\Delta k \sim T / \hbar v_{\mathrm{F}}\right)$ becomes sufficiently wide such that electrons fail to reach the constricted detector. Here, $c=(a+b) / 2$ is the characteristic propagation length. In addition, the ballistic nature is weakened by electron-phonon scattering. We therefore believe that the observed temperature dependence of the focusing peaks results from the thermally broadened momentum distribution and the reduced mean free path.

Below $40 \mathrm{~K}$, quasi-periodic oscillations appear, increasing near the focusing peak with a period of $\sim 2 \mathrm{~V}$ (Fig. $3 \mathrm{a}$ ). These oscillations are probably a result of quantum interference of refracted electron beams with different travel distances ${ }^{5}$. This results in spatial modulation of the current density near the focal point with a period of $2 \lambda_{\mathrm{F}}$ for $n \sim-1.2$. Given that the focal point moves by an amount $\Delta \delta=\left(\partial \delta / \partial V_{\mathrm{T}}\right) \Delta V_{\mathrm{T}}=-b \sqrt{V_{\mathrm{B}} / V_{\mathrm{T}}^{3}} \Delta V_{\mathrm{T}}$ with changing $\Delta V_{\mathrm{T}}$, the oscillation period $\Delta V_{\mathrm{T}}=2 \sim 3 \mathrm{~V}$ is estimated under the condition $\Delta \delta \sim 2 \lambda_{\mathrm{F}}$. Here, $\delta=2(b /|n|-a)$ is the focal position with respect to the detection constriction. According to the simple energy scale analysis, phase coherent oscillation survives up to a temperature of the order $\hbar v_{\mathrm{F}} / c \sim 10 \mathrm{~K}$, which is reasonably consistent with the observed temperature dependence. The data in Fig. 2a were taken at a finite temperature of $T=100 \mathrm{~K}$ to study electron focusing behaviour while suppressing this quantum interference. Another possible origin of the oscillation is a Fabry-Perot-type interference phenomenon of successively reflected electrons in the cavity of 


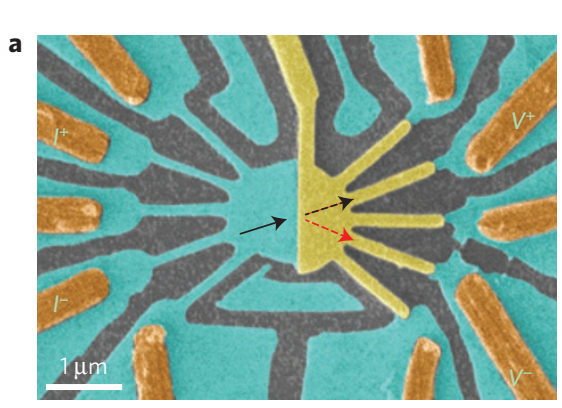

b

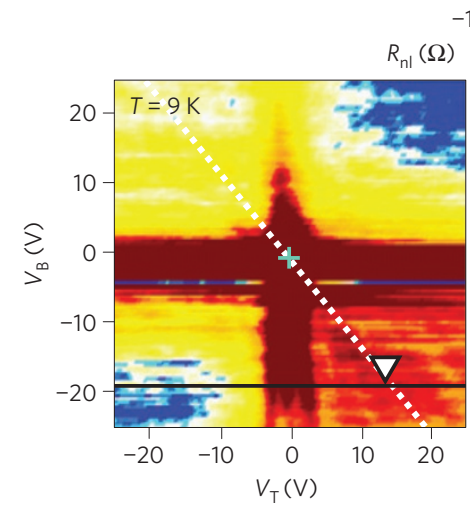

$-100$

50
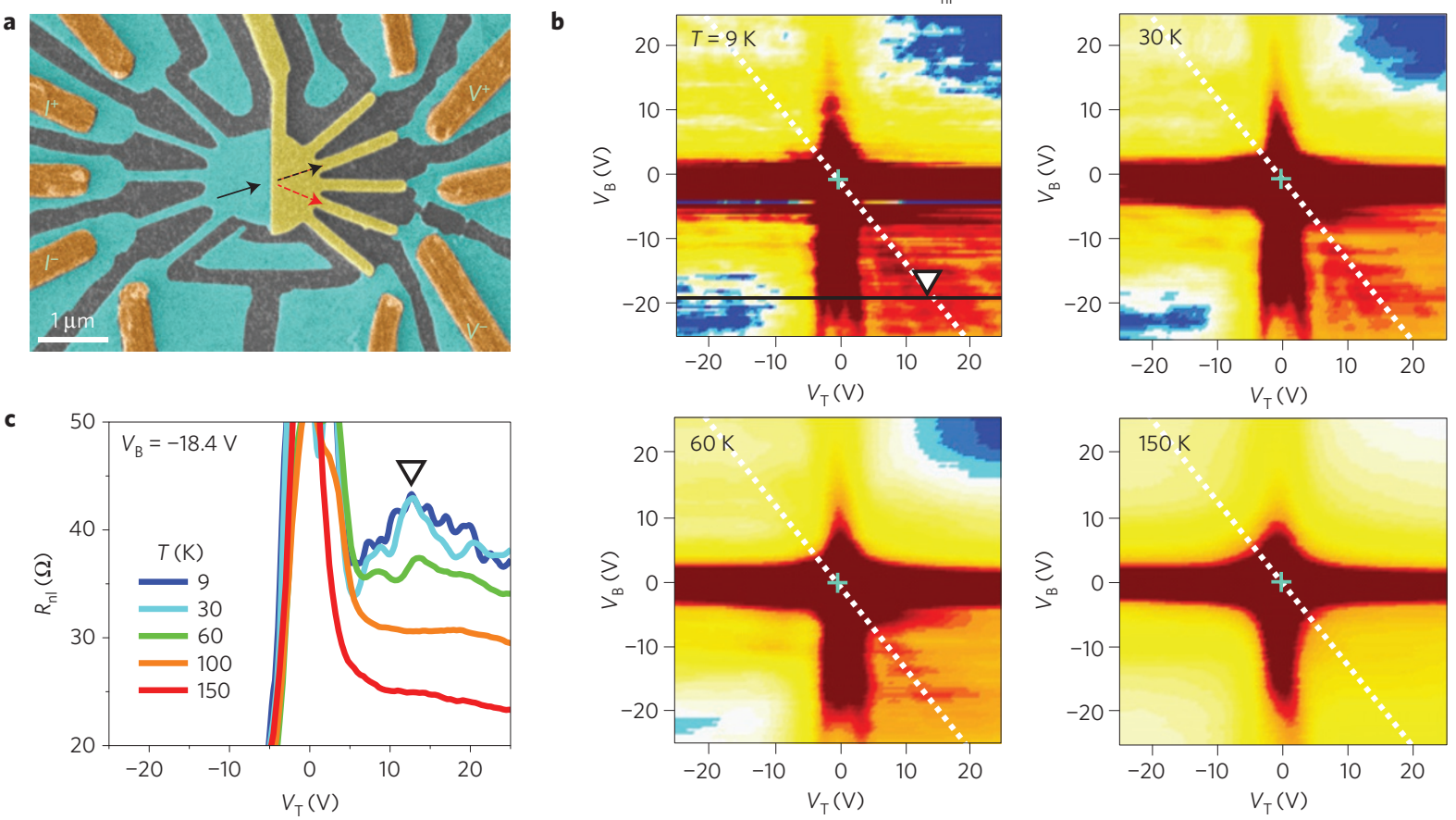

Figure 4 | Voltage measurement for negative refraction. a, Scanning electron microscopy (SEM) image of a non-local measurement device. While injecting a bias current $\left(I_{\text {bias }}\right)$ from $I^{+}$to $I^{-}$, a voltage difference $\Delta V=V^{+}-V^{-}$was measured. Injected electrons (black solid arrow) can be refracted with either a positive (black dotted arrow) or a negative (red dotted arrow) refractive index, depending on the top ( $V_{T}$ ) and bottom ( $V_{B}$ ) gate voltages.

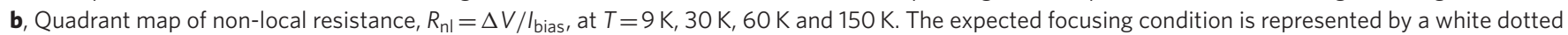
line. $\mathbf{c}, V_{\mathrm{T}}$ dependence of $R_{\mathrm{nl}}$ at $V_{\mathrm{B}}=-18.4 \mathrm{~V}$ (black line in $\mathbf{b}$ ) at various temperatures. The inverted triangle represents the position of the $R_{\mathrm{nl}}$ peaks.

the top-gated region. However, the expected Fabry-Perot period $\Delta V_{\mathrm{T}} \sim 0.4 \mathrm{~V}$ with a cavity size of $2 b$ deviates significantly from the measured period. Compared to a previous study with a diffusive back-gated region and wide cavity geometry ${ }^{17}$, the ballistic nature of the back-gated region and the narrow cavity of the Veselago lens in this study supplied more electrons along the normal incidence direction; the consequent lack of backscattering of those electrons leads to a less pronounced Fabry-Perot resonance. By applying a magnetic field, however, the Fabry-Perot oscillation can be enhanced, the period of which matches well with the expected value (see Supplementary Fig. 8).

Thus far, current measurements for verification of the electronic Veselago focusing through the graphene heterostructures have been discussed. To improve discrimination of the Veselago negative refraction signal from the large background, a voltage measurement scheme in a non-local geometry was adopted. Previously, this technique has been adopted to study graphene phenomena such as spin transport ${ }^{22}$, the spin Hall effect ${ }^{23}$ or, more recently, the valley Hall effect ${ }^{24}$. Our non-local device consisted of a single $\mathrm{p}-\mathrm{n}$ junction in circular ballistic graphene with multiple directional leads (Fig. 4a). The non-local resistance, $R_{\mathrm{nl}}=\left(V^{+}-V^{-}\right) / I_{\text {bias }}$, was measured as a function of $V_{\mathrm{T}}$ and $V_{\mathrm{B}}$. When two semicircular graphene layers had similar carrier density with the same polarity $\left(V_{\mathrm{T}} \sim V_{\mathrm{B}}\right)$, injected electrons (black arrow) were refracted with positive $n(\sim 1)$ and reached the electrode $V^{+}$(black dotted arrow). Electrons accumulated at the electrode $V^{+}$lowered the potential and resulted in a negative $R_{\mathrm{nl}}\left(V^{+}<V^{-}\right.$; blue-coloured regions in Fig. 4b), confirming the ballistic nature of the system at $T \leq \sim 60 \mathrm{~K}$. In contrast, in a bipolar regime with a similar carrier density $\left(V_{\mathrm{T}} \sim-V_{\mathrm{B}}\right)$ the $\mathrm{p}-\mathrm{n}$ junction deflected injected electrons towards the electrode $V^{-}$with a negative $n(\sim-1$; red dotted arrow), which reversed the sign of $R_{\mathrm{nl}}$ to positive. Long, directive guiding constrictions set the incidence angle $\left(\theta_{\mathrm{i}}=21^{\circ}\right)$ for the injected electrons and the refraction angle $\left(\theta_{\mathrm{r}}=-23^{\circ}\right)$ for the electrons to be refracted towards the electrode $V^{-}$. This gives a focusing condition of $V_{\mathrm{T}} / V_{\mathrm{B}}=-0.84$, or equivalently $n=-0.92$, represented by the dotted white line in Fig. $4 \mathrm{~b}$, along which $R_{\mathrm{nl}}$ is most strongly enhanced. In Fig. 4c, line cuts of $R_{\mathrm{nl}}$ as a function of $V_{\mathrm{T}}$ at a fixed $V_{\mathrm{B}}$ clearly show the enhancement of $R_{\mathrm{nl}}$ for $T \leq 60 \mathrm{~K}$, although small fluctuations are present through superposition of the quantum interference signal. A similar length scale analysis to that used above suggests that the focusing signal should persist up to $\sim 200 \mathrm{~K}$. However, the coincident disappearance of the enhanced $R_{\mathrm{nl}}$ in the bipolar regime and the negative $R_{\mathrm{nl}}$ in the unipolar regime above $100 \mathrm{~K}$ suggests that the focusing effect was limited by the ballisticity of the system.

Finally, we comment on the reproducibility of the results discussed above as well as on perspectives for improving this phenomenon. Two additional devices (one with current measurement and the other with non-local voltage measurement) reproduced essentially the same negative refraction behaviour discussed so far (see Supplementary Figs 9 and 10). Ballistic graphene heterostructures with sharp $\mathrm{p}-\mathrm{n}$ junctions enabled investigation of the unique electronic optics with a gate-tunable negative refractive index. One can improve the quality of graphene electronic optics by adopting readily available fabrication technologies, such as a superlattice top gate to collimate the injected electron beam ${ }^{25}$, a quantum point contact for the detector constriction $^{26}$, or mechanically cleaved graphite as a top gate electrode with atomically flat edges. Absorbers ${ }^{7}$ around the device edges can also help eliminate parasitic electrons and enhance focusing visibility. These efforts will lead to other interesting physics such as Klein tunnelling ${ }^{17}$, specular Andreev reflection with superconducting contacts ${ }^{27,28}$, or a perfect Cooper pair splitter ${ }^{29,30}$. With a long mean free path $(\sim 1 \mu \mathrm{m})$ at room temperature, due to very weak electron-phonon interactions at a large optical 
phonon energy, graphene is a promising material to provide novel components for electronic optics operating at high temperatures.

\section{Methods}

Methods and any associated references are available in the online version of the paper.

\section{Received 20 June 2015; accepted 3 August 2015;} published online 14 September 2015

\section{References}

1. Veselago, V. G. The electrodynamics of substances with simultaneously negative values of $\varepsilon$ and $\mu$. Sov. Phys. Usp. 10, 509-514 (1968).

2. Shalaev, V. M. Optical negative-index metamaterials. Nature Photon. 1, 41-48 (2007).

3. Schurig, D. et al. Metamaterial electromagnetic cloak at microwave frequencies. Science 314, 977-980 (2006).

4. Brunet, T. et al. Soft 3D acoustic metamaterial with negative index. Nature Mater. 14, 384-388 (2015).

5. Cheianov, V. V., Fal'ko, V. \& Altshuler, B. L. The focusing of electron flow and a Veselago lens in graphene p-n Junctions. Science 315, 1252-1255 (2007).

6. Geim, A. K. \& Grigorieva, I. V. Van der Waals heterostructures. Nature 499, 419-425 (2013).

7. Spector, J., Stormer, H. L., Baldwin, K. W., Pfeiffer, L. N. \& West, K. W. Electron focusing in two-dimensional systems by means of an electrostatic lens. Appl. Phys. Lett. 56, 1290-1292 (1990).

8. Huard, B. et al. Transport measurements across a tunable potential barrier in graphene. Phys. Rev. Lett. 98, 236803 (2007).

9. Williams, J. R., DiCarlo, L. \& Marcus, C. M. Quantum Hall effect in a gate-controlled $p-n$ junction of graphene. Science 317, 638-641 (2007).

10. Özyilmaz, B. et al. Electronic transport and quantum Hall effect in bipolar graphene $p-n-p$ junctions. Phys. Rev. Lett. 99, 166804 (2007).

11. Gorbachev, R. V., Mayorov, A. S., Savchenko, A. K., Horsell, D. W. \& Guinea, F. Conductance of $\mathrm{p}-\mathrm{n}-\mathrm{p}$ graphene structures with "Air-Bridge" top gates. Nano Lett. 8, 1995-1999 (2008).

12. Williams, J. R., Low, T., Lundstrom, M. S. \& Marcus, C. M. Gate-controlled guiding of electrons in graphene. Nature Nanotech. 6, 222-225 (2011).

13. Grushina, A. L., Ki, D.-K. \& Morpurgo, A. F. A ballistic pn junction in suspended graphene with split bottom gates. Appl. Phys. Lett. 102, 223102 (2013)

14. Rickhaus, P. et al. Ballistic interferences in suspended graphene. Nature Commun. 4, 2342 (2013).

15. Rickhaus, P. et al. Snake trajectories in ultraclean graphene p-n junctions Nature Commun. 6, 6470 (2015)

16. Stander, N., Huard, B. \& Goldhaber-Gordon, D. Evidence for Klein tunneling in graphene $p-n$ junctions. Phys. Rev. Lett. 102, 026807 (2009).

17. Young, A. F. \& Kim, P. Quantum interference and Klein tunnelling in graphene heterojunctions. Nature Phys. 5, 222-226 (2009).

18. Pendry, J. B. Negative refraction makes a perfect lens. Phys. Rev. Lett. 85, 3966-3969 (2000).
19. Cheianov, V. V. \& Fal'ko, V. I. Selective transmission of Dirac electrons and ballistic magnetoresistance of $n-p$ junctions in graphene. Phys. Rev. B 74, 041403 (2006)

20. Sajjad, R. N., Sutar, S., Lee, J. U. \& Ghosh, A. W. Manifestation of chiral tunneling at a tilted graphene $p-n$ junction. Phys. Rev. B 86, 155412 (2012).

21. Phong, B. T. Illumination for computer generated pictures. Commun. ACM 18, 311-317 (1975)

22. Tombros, N., Jozsa, C., Popinciuc, M., Jonkman, H. T. \& van Wees, B. J. Electronic spin transport and spin precession in single graphene layers at room temperature. Nature 448, 571-574 (2007).

23. Balakrishnan, J., Koon, G. K. W., Jaiswal, M., Castro Neto, A. H. \& Özyilmaz, B. Colossal enhancement of spin-orbit coupling in weakly hydrogenated graphene. Nature Phys. 9, 284-287 (2013).

24. Gorbachev, R. V. et al. Detecting topological currents in graphene superlattices. Science 346, 448-451 (2014).

25. Park, C.-H., Son, Y.-W., Yang, L., Cohen, M. L. \& Louie, S. G. Electron beam supercollimation in graphene superlattices. Nano Lett. 8, 2920-2924 (2008).

26. Nakaharai, S., Williams, J. R. \& Marcus, C. M. Gate-defined graphene quantum point contact in the quantum Hall regime. Phys. Rev. Lett. 107, 036602 (2011)

27. Beenakker, C. W. J. Specular Andreev reflection in graphene. Phys. Rev. Lett. 97, 067007 (2006).

28. Komatsu, K., Li, C., Autier-Laurent, S., Bouchiat, H. \& Guéron, S. Superconducting proximity effect in long superconductor/graphene/ superconductor junctions: From specular Andreev reflection at zero field to the quantum Hall regime. Phys. Rev. B 86, 115412 (2012)

29. Cayssol, J. Crossed Andreev reflection in a graphene bipolar transistor. Phys. Rev. Lett. 100, 147001 (2008).

30. Benjamin, C. \& Pachos, J. K. Detecting entangled states in graphene via crossed Andreev reflection. Phys. Rev. B 78, 235403 (2008).

\section{Acknowledgements}

We thank M. Kim, J. H. Lee and J. Lee for helpful discussions on the device fabrication. We thank R. N. Sajjad and A. W. Ghosh for fruitful discussions and critical reading of the manuscript. We also thank P. Kim for valuable comments. This work was supported by the National Research Foundation (NRF) through the SRC Center for Topological Matter (Grant No. 2011-0030046) and the GFR Center for Advanced Soft Electronics (Grant No. 2014M3A6A5060956)

\section{Author contributions}

G.-H.L. and H.-J.L. conceived the idea and designed the project. H.-J.L. supervised the project. G.-H.L. and G.-H.P. fabricated the devices. G.-H.L. performed the measurements. G.-H.L. and H.-J.L. analysed the data and wrote the manuscript.

\section{Additional information}

Supplementary information is available in the online version of the paper. Reprints and permissions information is available online at www.nature.com/reprints. Correspondence and requests for materials should be addressed to H.-J.L.

\section{Competing financial interests}

The authors declare no competing financial interests. 


\section{Methods}

Estimation of angular distribution of injected electrons at the port IN. To demonstrate the Veselago lensing of electrons, one needs to start with a wide angular distribution of injected electrons. According to the Huygens-Fresnel principle, a small constriction of width $w$ comparable to the wavelength $\lambda$ causes a plane wave to spread out hemispherically. A narrower constriction gives a wider spread, as depicted in Supplementary Fig. 1. Therefore, we have made narrow constrictions for the Veselago lens device. Quantitatively, we can consider it as a single slit problem in optics with an angular dependence of the intensity $I$ similar to Fraunhofer diffraction

$$
I(\theta)=I_{0}\left[\frac{\sin (\pi w \sin \theta / \lambda)}{(\pi w \sin \theta / \lambda)}\right]^{2}
$$

where $I_{0}$ is the maximum intensity and $\theta$ is the deviation angle from the centre. With $w=100 \mathrm{~nm}$ and $\lambda=60 \mathrm{~nm}(w / \lambda \sim 1.7)$, we expect a reasonably well spread angular dependence in the range from $-45^{\circ}$ to $+45^{\circ}$ (see Supplementary Fig. 2).

Device fabrication. BN was mechanically exfoliated on polypropylene carbonate (PPC) spun on a silicon (Si) substrate, peeled off and transferred to a Gel film (Gel-Pak, PF-30/17-X4) prepared on a glass slide. A BN/PPC/Gel-film stamp was used to make a stacked BN/graphene (G)/BN structure on a Si substrate covered with $300 \mathrm{~nm} \mathrm{SiO}$ by successively aligning and picking up the graphene and the basal $\mathrm{BN}$ (ref. 31). BN/G/BN stacks were etched by means of $\mathrm{CF}_{4}$ reactive ion etching using PMMA (Poly-methyl methacrylate) polymer as an etching mask. To improve graphene edge contacts with the metal electrode, we used the same PMMA polymer layer as an etching mask as well as a lift-off resist layer such that a freshly etched graphene edge is never contaminated by the polymer. To connect the top gate to the outer electrode, exposed graphene edges were passivated with a 50-nm-thick aluminium oxide layer, which prevents electrical shortage between the top gate electrode and the exposed graphene edge (see Supplementary Fig. 3).

Estimation of electrostatic potential profile of $p-n$ junction with local top gate. To estimate how sharp the $\mathrm{p}-\mathrm{n}$ junction potential profile would be, we performed numerical finite element analysis with the commercial COMSOL Multiphysics package. Supplementary Fig. 4a depicts the top gate structure with a BN dielectric layer on the graphene. We assumed the relative dielectric constant of $\mathrm{BN}$ $\varepsilon_{\mathrm{r}, \mathrm{BN}} \sim 3.9$, which has been estimated to be between 3 and 4 in an independent work ${ }^{32}$. The thickness of the top gate electrode was $h_{\text {gate }} \sim 50 \mathrm{~nm}$. The thickness of the BN layer was estimated to be $h \sim 14 \mathrm{~nm}$ from the ratio between top and bottom gate voltages of the Dirac point of the graphene, assuming the $\mathrm{SiO}_{2}$ bottom gate dielectric layer has a relative dielectric constant $\varepsilon_{\mathrm{r}_{\mathrm{SiO}}} \sim 3.9$ and thickness $h_{\mathrm{SiO}_{2}} \sim 300 \mathrm{~nm}$. Supplementary Fig. $4 \mathrm{~b}$ shows the results of calculated potential profiles at the $\mathrm{p}-\mathrm{n}$ junction with different $h$. The neutral potential point is always away from the edge $(\Delta L=0)$ of the top gate electrode owing to leakage of the electric field from the top gate electrode. As $h$ is decreased, this leakage is reduced such that the neutral potential point get closer to $\Delta L=0$ and the $\mathrm{p}-\mathrm{n}$ potential gets sharper. The characteristic sharpness of $d$ reaches as low as $12 \mathrm{~nm}$ when $h=15 \mathrm{~nm}$.

Classical particle trajectory simulation. We performed classical particle trajectory analysis to quantitatively explain the experimental observations of the focusing current. We assumed $N$ particles are injected into the port IN with a random initial angle $\theta_{\text {ini }}$ ranging from $-45^{\circ}$ to $+45^{\circ}$ and a random initial position within the width of the constriction $w_{\text {in }} \sim 100 \mathrm{~nm}$ (Supplementary Fig. 7a). At each $\mathrm{p}-\mathrm{n}$ junction, the refraction angle and the transmission probability $T$ were calculated using equations (1) and (2) in the main text with a given refractive index $n$ and specularity parameter $\alpha$ of Phong's model. Finally, we counted the number of electrons $\left(N_{\text {focus }}\right)$ which reached the constriction $\left(w_{\text {out }} \sim 100 \mathrm{~nm}\right)$ of the port OUT and calculated the probability, $p=N_{\text {focus }} / N$, for electrons to be refocused. Supplementary Fig. 7b shows the FWHM of the probability distribution of the error angle $\delta$ as a function of $\alpha$. Infinite $\alpha$ represents perfect specularity of the interface, whereas $\alpha=0$ means the refracted beam has a totally random angle. Supplementary Fig. $7 \mathrm{c}$ shows that the statistics become stable when $N$ is greater than $\sim 10,000$. Therefore, we used more than 10,000 particles in obtaining the probability for a given parameter set. In Supplementary Fig. 7d, a larger value of $\alpha$ gives a sharper focusing peak, which is consistently at $n \sim-1.2$, as we predicted in the main text.

\section{References}

31. Wang, L. et al. One-dimensional electrical contact to a two-dimensional material. Science 342, 614-617 (2013).

32. Young, A. F. et al. Electronic compressibility of layer-polarized bilayer graphene. Phys. Rev. B 85, 235458 (2012). 